\title{
Adsorption of Guanine, Guanosine, and Adenine at Electrodes Studied by Differential Pulse Voltammetry and Electrochemical Impedance
}

\author{
Ana Maria Oliveira-Brett,* Luís Antônio da Silva, and Christopher M. A. Brett* \\ Departamento de Química, Universidade de Coi mbra, 3004-535 Coi mbra, Portugal \\ Received J une 27, 2001. In Final Form: October 18, 2001
}

\begin{abstract}
Guanine, guanosine, and adenine have been el ectrochemically oxidized at a glassy carbon el ectrode in aqueous sol ution. Electrochemical impedance is shown to bea valuable probe of adsorption of the oxidation products on the el ectrode surface. Voltammetric and impedance studies at different concentrations have been carried out. Correlation between the results for the different compounds helps in understanding the adsorption process and the preconcentration properties of purinebases. I rradiation with ultrasound during recording of the impedance spectrum enabled adsorption to be avoided and the electrode process of the solution-soluble species to be investigated, and oxidation rate constants were calculated.
\end{abstract}

\section{Introduction}

It is of fundamental importance for understanding the function of DNA purine bases to understand their spontaneous adsorption on surfaces. For this reason, the el ectrochemical study of nucl eicacids and their adsorption on different types of el ectrodematerials has recently been of great interest. ${ }^{1-3}$ Most of the work has investigated either the reduction of the pyrimidine and purine bases on the mercury electrode $\mathrm{e}^{4-6}$ or their oxidation at glassy carbon el ectrodes. ${ }^{7,8}$ Electrochemically induced adsorption has usually been observed. Adsorption of guanine and guanosine on pyrolytic graphite el ectrodes has been fully characterized, and theimplicati ons for the determination of guanine in the presence of guanosine have been discussed. ${ }^{9} \mathrm{~A}$ similar behavior was found at glassy carbon electrodes. ${ }^{10}$

The electrochemical oxidation mechanism of guanine and adenine was investigated and follows a very similar mechanism to that for other purines..$^{11-17}$ It is $\mathrm{pH}$ dependent, and the electron transfer process occurs in consecutive steps with the formation of very strongly adsorbed oxidation products and di mers on the el ectrode

* To whom correspondence should be addressed. Telephone: +351-239-835295. Fax: +351-239-835295. E-mail: brett@ci.uc.pt.

(1) Palecek, E. In Topics in Bioel ectrochemistry and Bioenergetics; Milazzo, G., Ed.; Wiley: London, 1983; Vol. 5, p 65.

(2) Oliveira-Brett, A. M.; Matysik, F.-M. Bi oelectrochem. Bioenerg. 1997, 42, 111.

(3) Oliveira-Brett, A. M.; Matysik, F.-M.J . Electroanal. Chem. 1997, 429, 95.

(4) Dryhurst, G.; Elving, P. J . Talanta 1969, 16, 855

(5) Elving, P. J .; Pace, S. J .; O'Reilly, J . E. J . Am. Chem. Soc. 1973 $95,647$.

(6) Palecek, E. Electroanalysis 1996, 8, 1.

(7) Yao, T.; Musha, S. Bull. Chem. Soc. J pn. 1979, 52, 2307.

(8) Dryhurst, G. Talanta 1972, 19, 769.

(9) Dryhurst, G. Anal. Chim. Acta 1971, 57, 137.

(10) Yao, T.; Taniguchi, Y.; Wasa, T.; Musha, S. Bull. Chem. Soc. J pn. 1978, 51, 2937.

(11) Dryhurst, G.; Face, G. F. J . Electrochem. Soc. 1970, 117, 1259.

(12) Brett, C. M. A.; Oliveira-Brett, A. M.; Serrano, S. H. P. J . Electroanal. Chem. 1994, 366, 225.

(13) Subramanian, P.; Dryhurst, G.J . Electroanal. Chem. 1987, 244, 137.

(14) Dryhurst, G. J . Electrochem. Soc. 1969, 116, 1411.

(15) Dryhurst, G.; Elving, P.J .J . Electrochem. Soc. 1968, 115, 1014.

(16) Goya, R. N.; Dryhurst, G.J . Electroanal. Chem. 1982, 135, 75.

(17) Oliveira-Brett, A. M.; Piedade, J. A. P.; Serrano, S. H. P. Electroanalysis 2000, 12, 969. surface for both compounds. However, although the adsorbates were identified, ${ }^{14,15}$ their adsorption on the electrode surface has never been investigated.

The objective of this work was to study the adsorption of guanine, of its corresponding nucleoside, guanosine, of adenine, and of their oxidation products on a glassy carbon electrode. For this purpose, differential pulsevoltammetry and electrochemical impedancewereused. Understanding the adsorption process of these components of DNA is necessary toget insight intothebehavior of DNA-modified electrodes previously investigated using electrochemical impedance. ${ }^{18}$ This paper also demonstrates that sonoelectrochemical impedance is an effective technique for avoiding complications due to adsorbed material.

\section{Experimental Section}

Guanine, adenine, and guanosine (Sigma, > 99\% purity) were used as received without further purification. All other reagents were of analytical grade, and all solutions were prepared using Millipore Milli-Q ultrapure water (conductivity $<0.1 \mu \mathrm{S} \mathrm{cm}{ }^{-1}$ ). Stock solutions of the bases were prepared in $0.2 \mathrm{M}$ acetatebuffer at $\mathrm{pH}$ 4.5. The stock solution of guanosine $\left(1.5 \times 10^{-2} \mathrm{M}\right)$ was made by sl ow heating of the base in electrolyte until d ose to 100 ${ }^{\circ} \mathrm{C}$. Experiments were carried out in $\mathrm{pH}$ 4.5, $0.2 \mathrm{M}$ acetate buffer at $25 \pm 1{ }^{\circ} \mathrm{C}$.

A glassy carbon working el ectrode (Tokai GC 20) of diameter $3 \mathrm{~mm}$, a Pt wire counter electrode, and a $\mathrm{Ag} / \mathrm{AgCl}(3.0 \mathrm{M} \mathrm{KCl})$ reference electrode were placed in a one-compartment microcell using $200 \mu \mathrm{L}$ of solution.

Voltammograms were obtained using a PC-controlled PG STAT10 Autolab (EcoChemie) potentiostat/galvanostat with GPES 4.5 software. Differential pulse(DP) voltammograms were registered in the potential interval 0 to $+1.4 \mathrm{~V}$ versus $\mathrm{Ag} / \mathrm{AgCl}$, and the DP conditions were as follows: pulseamplitude, $50 \mathrm{mV}$; pulse width, $70 \mathrm{~ms}$; scan rate, $5 \mathrm{mV} \mathrm{s}^{-1}$.

Electrochemical impedance measurements were carried out using a Solartron 1250 frequency response analyzer, coupled to a Solartron 1286 electrochemical interface controlled by Zplot software; fitting of the spectra was doneby ZSI M CNLS software. A rms perturbation of $5 \mathrm{mV}$ was applied over thefrequency range $65 \mathrm{kHz}$ to $0.01 \mathrm{~Hz}$ with five frequencies per decade at chosen potentials in the range $0.0-1.4 \mathrm{~V}$ versus $\mathrm{Ag} / \mathrm{AgCl}$.

Sonoel ectrochemical impedanceexperiments werecarried out by irradiating the glassy carbon el ectrodewith ultrasound during the running of impedance experiments. A Vibra cell model 501

(18) Brett, C. M. A.; Oliveira-Brett, A. M.; Serrano, S. H. P. Electrochim. Acta 1999, 44, 4233. 


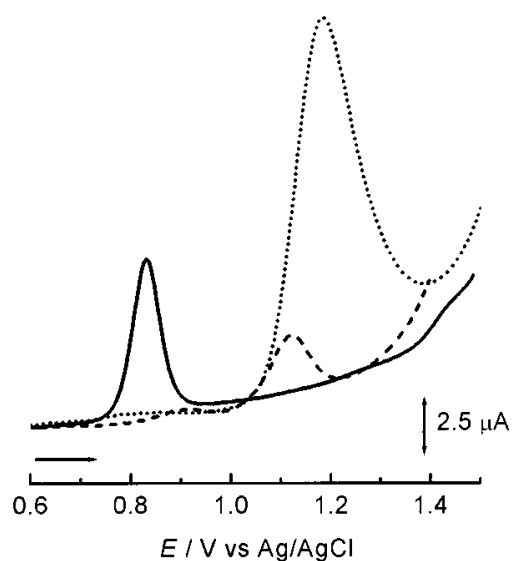

Figure 1. DP voltammograms for $(-) 3 \times 10^{-5} \mathrm{M}$ guanine, $(--) 5 \times 10^{-5} \mathrm{M}$ adenine, and $(\cdots) 5 \times 10^{-5} \mathrm{M}$ guanosine, in $\mathrm{pH} 4.5,0.2 \mathrm{M}$ acetate buffer: scan rate, $5 \mathrm{mV} \mathrm{s}^{-1}$; amplitude, $50 \mathrm{mV}$.

$20 \mathrm{kHz}$ sonichorn (Sonics \& Materials I nc.) with a $3 \mathrm{~mm}$ diameter Ti-tipped probewas employed, placed at a distance of $6 \mathrm{~mm}$ from theglassy carbon el ectrodesurface. Theelectrodes wereimmersed in a thermostated cell of $20 \mathrm{~cm}^{3}$ capacity.

\section{Results and Discussion}

Oxidation of Guanine. The oxidation of guanine occurs at $\sim 0.85 \mathrm{~V}$, as shown in the differential pulse voltammogram in Figure 1. A saturated solution of concentration $1 \mathrm{mM}$, well above the solubility limit for guanine, was prepared; the filtered supernatant was used for the vol tammetric experiments in Figure 1, the actual concentration in the electrolyte solution being $3 \times 10^{-5}$ M. ${ }^{2}$ The peak width at half-height of $\sim 70 \mathrm{mV}$ shows that the oxidation follows quasi-reversi ble electrode kinetics under the conditions employed. Since the first, ratedetermining, step of theoxidation involves two el ectrons, 7,8 a peak width of $45 \mathrm{mV}$ would be expected for reversible kinetics. In fact, guanine oxidation occurs in two consecutive steps. The first step is oxidation to 8-oxoguanine (8oxOG) followed by the one-electron oxidation of guanine dimers. ${ }^{13,17}$ The oxidation of 8-oxoG, corresponding to a reversi bletwo-el ectron/two-proton process, occurs at +0.55 $\mathrm{V}$, and oxidized 8-oxoG undergoes rapid hydrolysis. ${ }^{16,17}$ Soit does not interferewith themeasurements madehere. The guanine dimers formed during the oxidation of guanine are strongly adsorbed on the electrode surface and are reversibly oxidized.

Electrochemical impedance experiments offer an excellent way tofurther probetheadsorption phenomenon and theinfluence of base concentration and of potential. Thus, spectra were recorded at different applied potentials in the interval $0.0-1.0 \mathrm{~V}$ and for different concentrations. Figure2a shows compl ex planeimpedanceplots registered at different potentials for $3 \times 10^{-5} \mathrm{M}$ guanine in acetate buffer el ectrolyte, and Figure $2 \mathrm{~b}$ shows consecutivespectra recorded at an applied potential of $+0.85 \mathrm{~V}$ versus $\mathrm{Ag} /$ $\mathrm{AgCl}$, just before the current peak reaches its maximum value.

The various factors which influence the shape of the impedanceplots are now considered. Figure2a shows that, at values of potential below that necessary for guanine oxidation, theimpedance behavior is essentially capacitive modified by surface roughness effects, leading to a straight line of angle less than $90^{\circ}$. At $+0.80 \mathrm{~V}$-the beginning of the oxidation peak (as shown in Figure 1)-the chargetransfer reaction begins to havean influence on the shape of the spectrum. At $+0.85 \mathrm{~V}$ a high-frequency semicircle appears because of the charge-transfer reaction, and at
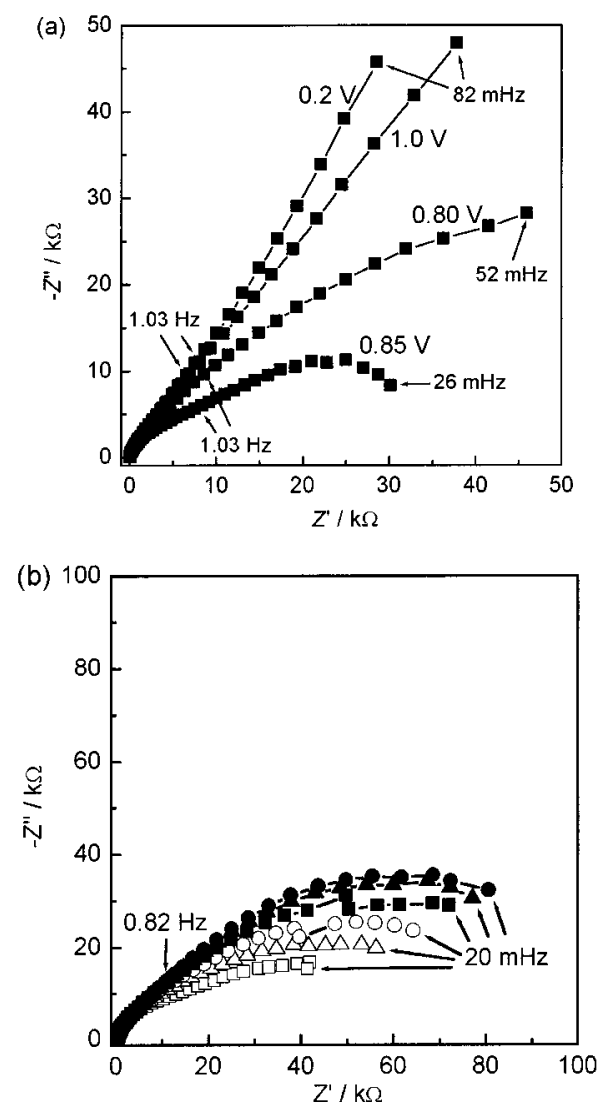

Figure 2. Complex plane impedance spectra for $3 \times 10^{-5} \mathrm{M}$ guanine in $\mathrm{pH}$ 4.5, $0.2 \mathrm{M}$ acetate buffer: (a) different applied potentials; (b) consecutive spectra at $+0.85 \mathrm{~V}$, from scan 1 ( $\square$ ) to scan $6(\mathbf{\bullet})$.

low frequency the behavior can beattributed torelaxation of the double layer due to reaction products adsorbed on the electrodesurface. Diffusion-limited oxidation at +1.0 $\checkmark$ leads to a straight line in the impedance plot. These phenomena cannot be observed by differential pulse voltammetry. From Figure $2 \mathrm{~b}$ it can be seen that there is an increase in the size of the semicircle with successive scans, showing the effect of surface blocking on the measured charge-transfer resistance.

The spectra were fitted to the electrical equivalent circuit shown in Figure 3a. The charge-transfer reaction is represented by a charge-transfer resistance, $R_{c t}$, in parallel with a constant phase element $C P E_{1}$ with characteristics of a capacitance, to take into account the frequency dispersion often related directly to electrode roughness, ${ }_{19}^{19}$ which is normally found for solid el ectrodes. The low frequency double layer relaxation is described by the $R_{2} / C P E_{2}$ parallel combination in series with $R_{c t}$. The CPE exponents found here had values between 0.90 and 0.80. $R_{\Omega}$ represents the ohmic resistance of the cell. At potentials less than necessary for guanine oxidation, $R_{\mathrm{ct}}$ and $R_{2} / C P E_{2}$ were removed from the ectrical equival ent circuit. An example of the fitting procedure is shown in the Bode plot of Figure 3b.

Taking surface roughness into account, as described above, values of interfacial capacitance were found between 5 and $12 \mu \mathrm{F}$, the lowest values being at potentials d osetothat for oxidation of guanine, as would be predicted. Values of $\mathrm{CPE}_{2}$ were around $0.5 \mathrm{mF} \mathrm{cm}^{-2}$, an order of magnitude expected for adsorbed species.

Fitted values of $R_{c t}$ are given in Table 1, together with the corresponding values of oxidation peak current, $I_{p}$,

(19) De Levie, R. Electrochim. Acta 1964, 9, 1231. 
(a)
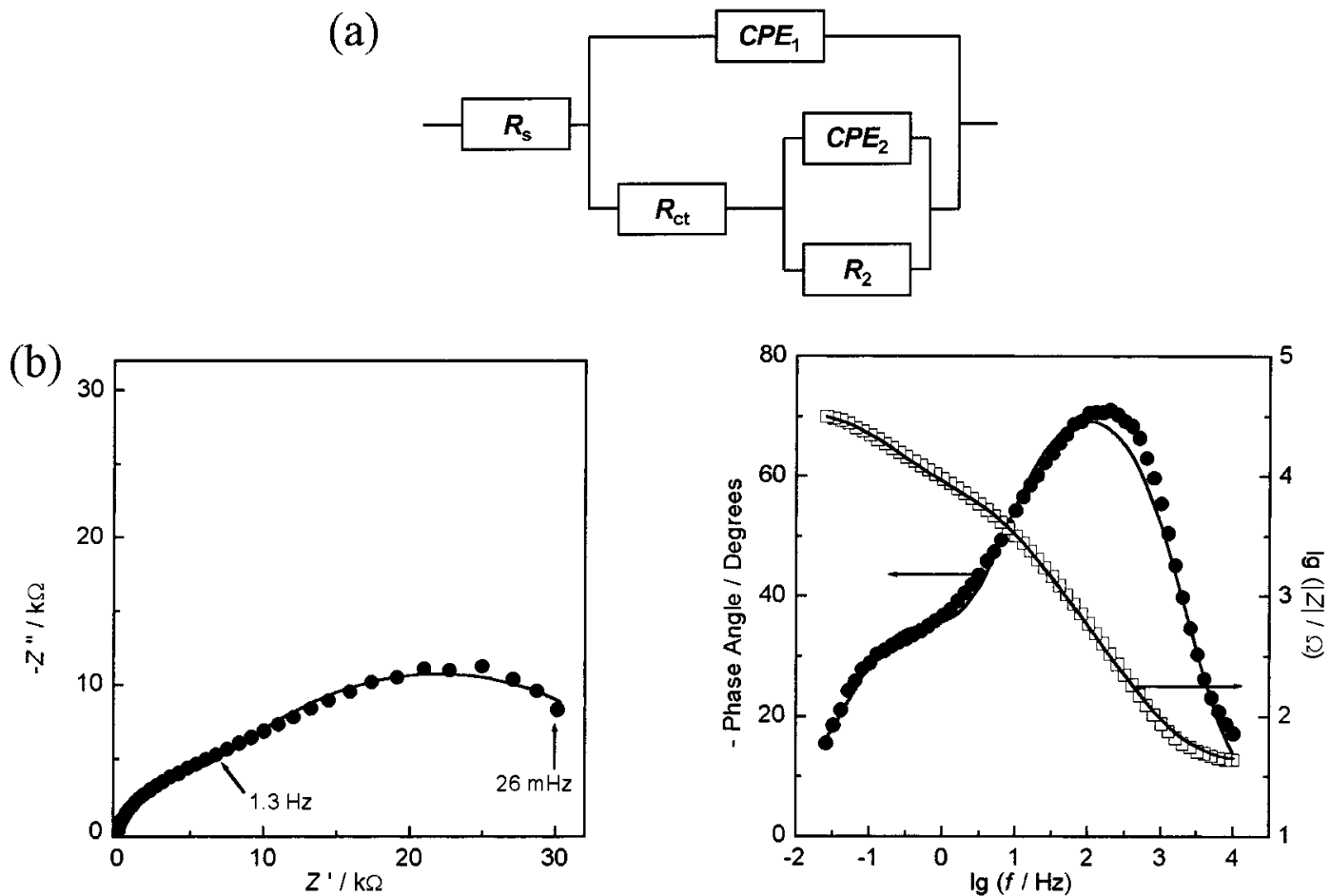

Figure 3. Fitting of impedance spectra: (a) Equivalent circuit. (b) Fitting at $0.85 \mathrm{~V}$ for $3 \times 10^{-5} \mathrm{M}$ guanine; data from Figure 2a. The solid line shows the result of fitting to the equivalent circuit.

Table 1. Guanine Oxidation Peak Current, I , from DPV and $\mathbf{R}_{\mathrm{ct}}$ from Impedance Spectra at $+0.85 \mathrm{~V}$ for Consecutive Scans, in pH 4.5, 0.2 M Acetate Buffer

\begin{tabular}{ccc}
\hline scan no. & $\mathrm{I}_{\mathrm{p}} / \mu \mathrm{A}$ (differential pulse) & $\mathrm{R}_{\mathrm{ct}} / \mathrm{k} \Omega$ (impedance) \\
\hline 1 & 6.39 & 24.2 \\
2 & 6.42 & 32.1 \\
3 & 6.39 & 38.0 \\
4 & 6.51 & 41.8 \\
5 & 6.66 & 45.2 \\
6 & 6.85 & 47.8
\end{tabular}

from differential pulse voltammetry. Comparison shows that both $R_{c t}$ and $I_{p}$ values increase with scan number. In differential pulsevoltammetry, the peak current increases by $\sim 3 \%$ and $R_{c t}$ increases by $\sim 90 \%$ after six scans. I fact, the val ue of $I_{p}$ is more or less constant over the first three scans and then begins to increase slightly. This can be attributed to the contribution from strongly adsorbed guaninedimers, ${ }^{14}$ formed on the el ectrodesurface between guanine and the adsorbed guanine oxidation products which slowly cover the surface. It was verified that after 20 scans the peak current begins to drop. However, each impedance experiment takes approximately $10 \mathrm{~min}$ and is conducted at a fixed value of applied potential, where an electrode reaction is occurring all the time. Therefore, theinfluence of adsorption of oxidation products blocking the surface is seen al ready in the second frequency scan.

To removetheeffect of blocking by adsorption products, impedance spectra were recorded while irradiating the electrode surface with ultrasound, the purpose being to exploit thecleaning effect of ultrasound as well as prevent blocking of the el ectrode surface. Several different ultrasound power levels were tried, to ascertain what was the minimum power level necessary for the cleaning effect to operate while minimizing the temperature perturbation. Second, experiments were done under irradiation after recording of spectra under silent conditions several times consecutively totest reproducibility. An exampleis shown in Figure 4. Thediameter of the high-frequency semicircle is $R_{c t}=5.1 \mathrm{k} \Omega$, significantly lower than the values

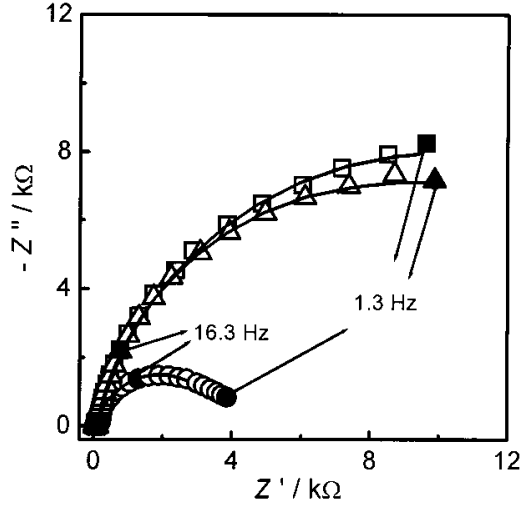

Figure 4. I mpedance spectra of $3 \times 10^{-5} \mathrm{M}$ guaninein $\mathrm{pH} 4.5$, $0.2 \mathrm{M}$ acetate buffer at $0.85 \mathrm{~V}:(\square)$ before application of ultrasound; $(O)$ with ultrasound power $=135 \mathrm{~W} \mathrm{~cm}^{-2} ;(\triangle)$ after ultrasound.

cal culated in theabsence of ultrasound. Assuming a simple electrode process, the equation

$$
\mathrm{R}_{\mathrm{ct}}=\frac{\mathrm{RT}}{(\mathrm{nF})^{2} \mathrm{Ak_{0 } \mathrm { C } _ { \infty }}}
$$

can be employed in order to calculate the standard rate constant, $\mathrm{k}_{0}$, from the high-frequency part of the spectra, where $A$ is the el ectrode area, $n=2$, and all other symbols have their usual meanings. A value for thestandard rate constant of $6.21 \times 10^{-3} \mathrm{~cm} \mathrm{~s}^{-1}$ is obtained.

Oxidation of Adenine. A differential pulse voltammogram for adenineis in $\mathrm{Figure}$ l and shows theoxidation occurring at $+1.1 \mathrm{~V}$ versus $\mathrm{Ag} / \mathrm{AgCl}$, and the greater peak width at half-height than that for guanine demonstrates that the oxidation reaction is slower. It occurs in three steps, and one of the oxidation products is adenine dimers. ${ }^{14,15}$ Adenineadsorbs very strongly on theel ectrode surface, compared to the adsorption of the oxidation products and the dimers. The more positive oxidation 
(a)

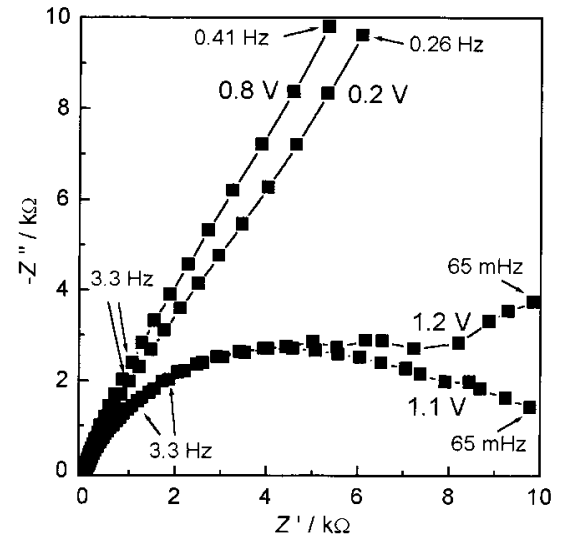

(b)

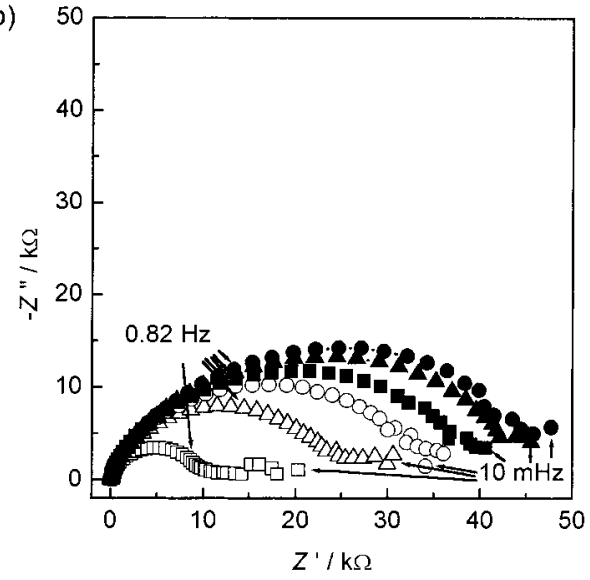

Figure 5. Complex plane impedance spectra for $1 \times 10^{-3} \mathrm{M}$ adenine in pH 4.5, 0.2 M acetate buffer: (a) different applied potentials; (b) consecutive spectra at $1.1 \mathrm{~V}$, from scan $1(\square)$ to scan $6(\bullet)$.

potential than that of guanine is due to the difference in chemical structure. Additionally, themore polar structure also means that, unlike the case of guanine, there is no problem of solubility in acetate buffer. At much higher concentrations of $1 \mathrm{mM}$, after the first scan the values of current diminish drastically, although the way in which blocking of the electrode surface occurs is qualitatively similar to that of guanine.

Complex planeimpedance spectra recorded at different applied potentials are shown in $\mathrm{F}$ igure $5 \mathrm{a}$, and theresults of consecutivescans for thesameconcentration are shown in Figure $5 b$. Once again, as for guanine, there is a capacitive behavior before the oxidation reaction begins and then a charge-transfer semicircle appears. However, thesecond semicircleduetorelaxation of the doublelayer, which was pronounced for guanine, is not so for adenine. Successive measurements at $+1.1 \mathrm{~V}$ show a similar variation with scan number as that for guanine. The variations of current and of $R_{c t}$ are shown in Table 2 and can be compared with the values in Table 1 for guanine. Comparatively speaking, thesurfaceis blocked by adenine much faster, which can be ascribed to its greater polarity. The variation of impedance spectra for different adenine concentrations is not large (Figure 6 and inset), which demonstrates that saturation adsorption occurs readily and on a time scale less than that of the impedance experiment.

Once again, the cleaning effect of ultrasonic radiation leads to a reduction in the high-frequency semicircle. Values of $8 \mathrm{k} \Omega$ are obtained, and this corresponds to a standard rate constant of $1.19 \times 10^{-4} \mathrm{~cm} \mathrm{~s}^{-1}$ for a twoelectron process.

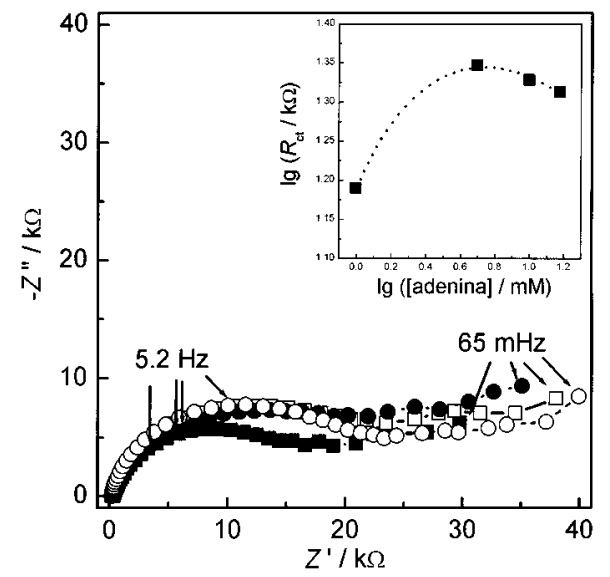

Figure 6. Complex plane impedance spectra for different concentrations of adenine at an applied potential of $+1.2 \mathrm{~V}$ : (ㅁ) $1 \times 10^{-3}$; (口) $5 \times 10^{-3}$; (○) $10 \times 10^{-3}$; (O) $15 \times 10^{-3} \mathrm{M}$. The inset is a plot of $\mathrm{R}_{\mathrm{ct}}{ }^{-1}$ versus adenine concentration.

Table 2. Adenine Oxidation Peak Current, $I_{p}$, from DPV and $\mathbf{R}_{\mathrm{ct}}$ from Impedance Spectra at $+\mathbf{1 . 1} \mathrm{V}$ for Consecutive Scans, in pH 4.5, 0.2 M Acetate Buffer

\begin{tabular}{ccc}
\hline scan no. & $\mathrm{I}_{\mathrm{p}} / \mu \mathrm{A}$ (differential pulse) & $\mathrm{R}_{\mathrm{ct}} / \mathrm{k} \Omega$ (impedance) \\
\hline 1 & 78.0 & 9.8 \\
2 & 12.0 & 22.2 \\
3 & 11.7 & 31.6 \\
4 & 10.0 & 37.5 \\
5 & 10.2 & 43.0 \\
6 & 10.9 & 46.7
\end{tabular}

Oxidation of Guanosine. Although guanine and guanosine have similar structures, the potentials necessary for their electro-oxi dation are different. As shown by the DPV of guanosine in Figure 1, guanosine oxidizes at $\sim 1.1 \mathrm{~V}$ in acetate buffer compared to $\sim 0.85 \mathrm{~V}$ for guanine. This difference can be attributed to the influence of the ribose group in making the removal of protons and electrons from the nitrogen base more difficult via inductive effects. However, the values of current in subsequent scans decrease, as in the case of adenine, owing to adsorption of oxidized guanosine on the surface of the electrode. Thevariation of peak current with concentration for the first and second DPV scans, at high concentrations, shows a pronounced reduction in current duetoadsorption. There is a saturation effect on increasing the concentration, which can be ascribed to blocking the electrode surface.

The various factors which influence the shape of the impedance pl ots areshown in Figure 7. This demonstrates that, as for guanine, at values of potential below those necessary for guanosineoxidation, theimpedancebehavior is essentially capacitive modified by surface roughness effects. At $+1.0 \mathrm{~V}$-thefoot of theoxidation peak in Figure 1-thecharge-transfer reaction begins to havean influence on the shape of the spectrum. From Figure 8 it can be seen that there is a decrease in the size of the semicircle with increasing concentration, the excess of reagent at the surface facilitating electron transfer.

In further experiments, impedance spectra were recorded sequentially at $+1.1 \mathrm{~V}$ for a guanosine concentration of $1.0 \times 10^{-3} \mathrm{M}$, and these demonstrate, more conclusively, how adsorption products block the el ectrode surface For the first two scans, two time constants are evident, as discussed above. They then become fused together and the semicircles become more depressed. Values of $R_{c t}$ were calculated and are illustrated in Table 3. It was verified that theincreasein $R_{\mathrm{ct}}$ was much greater for the highest concentrations of guanosine tested. 


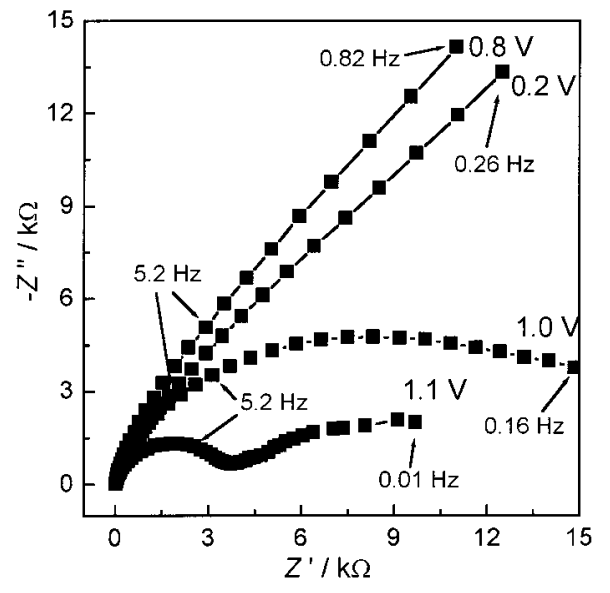

Figure 7. Complex plane impedance spectra for $1 \times 10^{-3} \mathrm{M}$ guanosine in $\mathrm{pH}$ 4.5, $0.2 \mathrm{M}$ acetate buffer at different applied potentials.

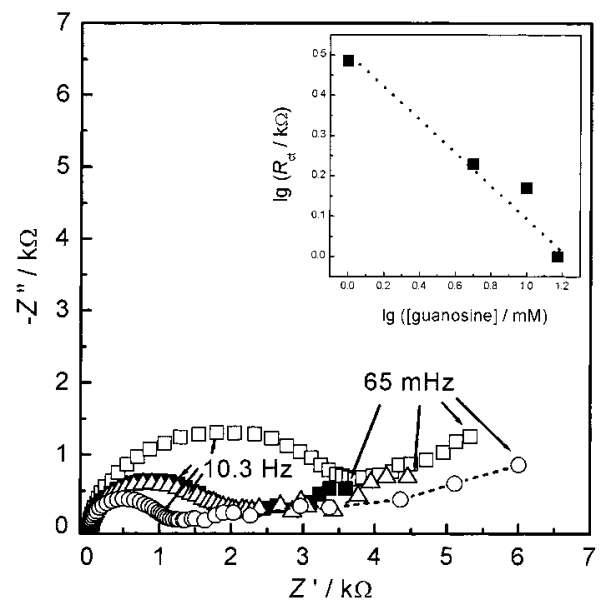

Figure 8. Complex plane impedance spectra for different concentrations of guanosine at an applied potential of $1.1 \mathrm{~V}$ : () $1 \times 10^{-3}$; (口) $5 \times 10^{-3} ;(\triangle) 10 \times 10^{-3}$; (O) $15 \times 10^{-3} \mathrm{M}$. The inset is a plot of $R_{c t}{ }^{-1}$ versus guanosine concentration.

Taking surface roughness into account, as described previously, values of capacitance were found between 5 and $12 \mu \mathrm{F}$, the lowest values being at potentials close to that for oxidation of guanosine, as would be predicted.

Application of ultrasound, as before, leads to a smaller and constant value of the charge-transfer resistance and is successful in removing adsorbed species from the electrode surface. Standard rate constants from sonoel ectrochemical impedance spectra areagain on theorder of $1.0 \times 10^{-4} \mathrm{~cm} \mathrm{~s}^{-1}$.

Comparative Remarks. Comparison of the three bases shows that adsorption is least for guanosine, as reflected by thelower values of charge-transfer resistance,
Table 3. Values of $\mathbf{R}_{\mathrm{ct}}$ from Impedance Spectra of Guanosine at $+1.1 \mathrm{~V}$ for Consecutive Scans, in pH 4.5, 0.2 M Acetate Buffer

\begin{tabular}{ccccc}
\hline & \multicolumn{4}{c}{$\mathrm{R}_{\mathrm{ct}}$ for the following values of [guanosine]/mM } \\
\cline { 2 - 5 } scan no. & \multicolumn{1}{c}{1} & \multicolumn{1}{c}{5} & 10 & 15 \\
\hline 1 & 3.0 & 2.4 & 2.2 & 1.6 \\
2 & 6.2 & 8.2 & 12.0 & 16.0 \\
3 & 8.1 & 15.2 & 22.4 & 29.1 \\
4 & 9.8 & 27.9 & 31.1 & 42.2 \\
5 & 12.0 & 33.8 & 34.2 & 56.8 \\
6 & 13.2 & 37.0 & 37.0 & 65.5
\end{tabular}

and must be due to the presence of the sugar group. Adenine and guanine show similar adsorption characteristics. In all cases, the application of ultrasound leads to removal of the adsorbed species and the values of oxidation rate constant are similar. It suggests that the strengths of adsorption of the DNA bases on the glassy carbon surface are similar. This can have important implications for the preparation of el ectrochemical DNA biosensors.

\section{Conclusions}

Differential pulse voltammetry and electrochemical impedance have demonstrated that guanine, adenine, guanosine, and their oxidation products are electrochemically adsorbed on glassy carbon electrode surfaces, and these behaviors show a concentration dependence. The dependenceof guanineadsorption on concentration is least evident in thesedata, owing to its low solubility in aqueous acetate buffer solution. Adsorption causes an increase in $R_{c t}$ from electrochemical impedance and a decrease in oxidation current. Thetimescale of adsorption is brought into evidence by comparison of differential pulse voltammetric and impedance results.

It is shown that irradiation by ultrasound during the recording of impedance spectra does not interfere with the measurements and removes adsorbed products, thus being a promising avenue for the measurement of rate constants without the influence of adsorption.

Acknowledgment. Financial support from Fundação para a Ciência eTecnologia (FCT) project PRAXIS/P/AGR/ $12205 / 1998$, POCTI (cofinanced by the European Community fund FEDER), ICEMS (Research Unit 103), and European Projects ERB-ICT15-CT960804 and QLK32000-01311 is gratefully acknowledged. 